\title{
Rash in an elderly woman presenting with collapse
}

\author{
C Prajapati, BS Phillips, MJ Bradshaw
}

An 87-year-old woman was brought to the Accident and Emergency department with a history of sudden loss of consciousness. On examination she was confused and had Glasgow Coma Scale of 9. She was hypothermic $\left(35^{\circ} \mathrm{C}\right)$, normotensive, in sinus rhythm and had a left hemiparesis. Her initial investigations, including chest X-ray and an electrocardiogram (ECG) were normal. After her transfer to the admitting ward her Glasgow Coma Scale improved to 14. On the following day she suddenly became pale and hypotensive and a cardiac monitor showed bradycardia with sinus arrest. She responded to resuscitation. Over the next 24 hours her left hemiparesis resolved completely but she became oliguric and developed bruising in both flanks. A further ECG showed ST segment depression in the inferior limb leads. Her blood tests revealed mild anaemia, abnormal clotting profile, impaired renal function and raised cardiac enzymes. Serum and urinary amylase were normal. Fresh frozen plasma was given and her urinary output improved with diuretics. Her clotting profile and renal function improved. Her condition remained stable for the next two days but on the fifth day of her admission she suffered a cardiac arrest and died.

Table Laboratory profile

Department of
Medicine for the
Elderly, Brighton
General Hospital, Elm
Grove, Brighton BN2
3EW, UK
C Prajapati
BS Phillips
MJ Bradshaw

Accepted 3 January 1996

\begin{tabular}{llllll}
\hline & Day 1 & Day 2 & Day 3 & Day 4 & Day 5 \\
\hline Haemogolobin (g/dl) & 11.9 & 10.9 & 10.1 & 10.4 & 9.1 \\
Platelets $\left(\times 10^{9} / \mathrm{l}\right)$ & 157 & 28 & 30 & 49 & 293 \\
INR & - & 2.0 & 1.6 & 1.5 & - \\
Fibrin degradation products & - & 40 & - & - & - \\
Creatinine $(\mu \mathrm{mol} / \mathrm{l})$ & 142 & 230 & 202 & 156 & 130 \\
Serum amylase (IU/l) & 880 & 850 & - & - & - \\
Urinary amylase (mmol/24 h) & - & $<30$ & - & - & - \\
Creatine kinase (IU/l) & 103 & 285 & 369 & 169 & - \\
Aspartate aminotransaminase (IU/l) & 164 & 1661 & 1752 & 837 & 32 \\
Lactate dehydrogenase (IU/l) & 990 & 5300 & 5307 & 1906 & - \\
\hline
\end{tabular}

\section{Questions}

1 What haematological complication did the patient develop?

2 What was the underlying diagnosis? 


\section{Answers}

QUESTION 1

The patient had developed disseminated intravascular coagulation. This diagnosis was made in view of thrombocytopenia, anaemia, raised INR, fibrin degradation products and renal impairment.

\section{QUESTION 2}

The underlying diagnosis was dissection of the ascending aorta. Dissection of the thoracic aorta commonly involves the ascending or arch of the aorta with variable clinical presentations depending upon the site of the dissection. Collapse, chest pain (which may radiate through to the back), and sudden neurological deficit are common presentations. In elderly patients, lack of history and silent presentation can cause diagnostic difficulties.

In our patient, there was no evidence of cerebral infarct or haemorrhage at post mortem. There was no evidence of myocardial infarction but severe atheroma was found in the left anterior descending and right coronary artery. There was $500 \mathrm{ml}$ of fresh clotted blood in the pericardial sac. There was a dissection of the aortic arch starting at the orifice of the right brachiocephalic artery extending proximally to coronary ostei and the root of the aorta which communicated with the pericardial sac. Occlusion by dissection of the right brachiocephalic artery would explain the transient hemiparesis and depression of consciousness. Occulsion of the right coronary artery would explain the transient sinus arrest, bradycardia, hypotension, ST segment changes in the inferior leads and elevated transaminase levels.

\section{Discussion}

Disseminated intravascular coagulation is a condition arising from activation of coagulation system which overwhelms the controlling system leading to generalised coagulation in

1 Chen WJ, Chen J, Lei MH. Dissecting aneurysm of the aorta associated with local consumption coagulopathy. Taiwan I Hsueh Hui Tsa Chih 1989; 88: 1167-70.

2 Disdier P, Harle JR, Ailluad MF, et al. Coagulation intravasculaire disseminee et dissection aortique chronique. (Disseminated intravascular coagulation and chronic aortic dissection). Ann Cardiol Angeiol (Paris) 1991; 40: 547-9.
Conditions associated with disseminated intravascular coagulation

- infection: septicaemia, viral infection, malaria

- obstetric: pre-eclampsia, abruptio placentae, intra-uterine foetal death, amniotic fluid emboli

- cardiovascular: shock, aortic aneurysm, giant haemangioma, vasculitis

- major trauma

- malignancy

- heat stroke

- snake bites

the circulation. It has been associated with diverse groups of clinical conditions (box). Only a few cases of association of disseminated intravascular coagulation and aortic dissection have been described in the literature. ${ }^{1-3} \mathrm{La}-$ boratory tests indicating disseminated intravascular coagulation include thrombocytopenia, increased fibrin degradation products, and prolonged prothrombin and partial prothrombin time.

Acute disseminated intravascular coagulation with bleeding tendency requires treatment with fresh frozen plasma as a source of coagulation factors. If the fibrinogen level is markedly depressed cryoprecipitate can be given. Platelets are only given if the count falls below $80 \times 10^{9} / 1$. In chronic disseminated intravascular coagulation, heparin is given, pending a definitive treatment.

\section{Final diagnosis}

Disseminated intravascular coagulation associated with aortic dissection.

Keywords: disseminated intravascular coagulation, aortic dissection

3 Mamiya S, Endo Y, Miura AB, et al. Disseminated intravascular coagulation accompanying thoracic and abdominal aortic aneurysm; report of three cases. $f_{p n ~} \mathcal{f} \mathrm{Med}$ 1988; 27: $91-5$. 